\title{
REVIEW
}

\section{Clinical decision units in the emergency department: old concepts, new paradigms, and refined gate keeping}

\section{T B Hassan}

Emerg Med J 2003;20:123-125

This review looks at the background of clinical decision units/observation units and their role in the management of patients in the emergency department.

$\mathrm{T}$ he inexorable pressures on accident and emergency departments (EDs) in the UK to deliver high quality clinical care and simultaneously meet external time performance standards has led to the need for radical change. ${ }^{2}$ In the midst of this, hospital congestion and "exit blocks" have continued to compromise the ability of departments to be able to function and meet these needs. ${ }^{3}$ Much time and effort has been spent convincing relevant stakeholders that there is a systems problem to solve. ${ }^{4}$ Hospitals are complex organisations where inefficiency in one area can have a significant impact on another. A variety of models have been developed to manage these systems particularly for patients requiring admission to the hospital bed base ${ }^{5}$ but with varying success.

Consultants in emergency medicine have been traditionally responsible for short stay observation units (OU) within their departments. These ward areas cater for certain categories of patients with surgical, medical, and/or psychosocial needs who can be discharged within 24-48 hours. ${ }^{6}$ They have tended to be condition specific but tailored to local needs and interests or more often, lack of alternative pathways within the hospital. ${ }^{7}$ Although the concept of the OU is considered good practice especially in minimising clinical risk, there has been little progress to further develop and improve the function of these units in UK practice.

Experience from the United States suggests that staff in the ED, given adequate resources could be responsible for managing a range of other conditions on OUs as well. This increases the efficiency and effectiveness of the ED in better gatekeeping of the medical bed base. More importantly an OU can have a significant impact in minimising clinical risk for certain occult life threatening conditions thus protecting the institution from damaging litigation. ${ }^{8}$

The implementation of the Reforming emergency care strategy ${ }^{2}$ is an opportunity for consultants in emergency medicine in the UK to re-appraise the functions of their OUs or potentially to develop such units within their departments in order to better manage certain categories of patients. This article aims to review the evidence for the use of OUs or clinical decision units (CDUs) as they are synonymously (and more dynamically) termed and define their potential for UK practice.

\section{HISTORICAL BASE}

Historically, Dallos and Mouzas ${ }^{9}$ were among the first in the UK to describe the work of their two A\&E observation wards in London that dealt with a range of medical and surgical conditions. These included chest pain, syncope, epilepsy, abdominal pain, head injury, and a variety of orthopaedic injuries as well as patients requiring psychiatric and social support. The median length of stay was reported as one day and at Whipps Cross the subsequent admission rate to the in-hospital bed base was $13 \%$. The model allowed certain groups of patients to be observed and investigated by A\&E staff before appropriate disposal and optimised the referral to and use of the in-hospital bed base.

Progress in the UK since the early 1980s has remained fragmented. In 1998 a comprehensive study of existing practices identified that only 95 A\&E departments out of 260 possessed short stay beds. ${ }^{7}$ They were used for a variety of conditions that included minor head injury, alcohol and drug intoxication, psychosocial care, soft tissue infections, and deliberate self harm or self poisoning. Much of this traditional practice derives from original recommendations on the role of short stay wards in A\&E departments made by the Joint Consultants Committee in $1978 .{ }^{10}$

More recently, some centres in the UK have developed chest pain evaluation units to specifically target the important group of patients with a symptom complex suggestive of a possible acute coronary syndrome. ${ }^{11}{ }^{12}$ These strategies, based in the ED have been proved to be safe and efficient. Their cost effectiveness in UK emergency departments is still to be reported but evidence from the United States strongly suggests that they will also prove to be more economical than standard in-hospital care. ${ }^{13}{ }^{14}$

In the United States, similar success with the development of the observation medicine concept was originally described by Landers $\mathrm{et}^{\mathrm{l}} \mathrm{l}^{15}$ and Diamond et $a^{16}$ in the 1970s with similar workload patterns and throughput. It was however the late 1980s before provision of services for OUs became standardised and the first practice guidelines were issued by the American College of Emergency Physicians. ${ }^{17}$ Subsequent debate and discussion refined the concept with development of a curriculum $^{18}{ }^{19}$ and complex re-structuring of the financial re-imbursements in the US needed for use of observation beds. ${ }^{8}$ The main emphasis has been in development of chest pain evaluation

Abbreviations: ED, emergency department; $\mathrm{OU}$ observation unit; $C D U$, clinical decision unit 
although protocols for a range of other conditions with a high likelihood of rapid discharge ( $<24$ hours) have also been developed. Interestingly, despite all this, progress in the US in developing OUs has remained slow. In 2000, a survey of academic departments of emergency medicine revealed that only $37 \%$ had a functioning OU. ${ }^{20}$ Reasons for this are considered to be related chiefly to the problems with financial re-imbursement in the complex healthcare system in the US. ${ }^{21}$

\section{EVIDENCE OF BENEFIT}

Evidence of a beneficial impact using ED based OUs for certain groups of patients instead of in-hospital facilities is steadily growing. The greatest experience thus far has been for chest pain evaluation. This is related to its high frequency as a condition presenting to the ED, the poor diagnostic performance of initial clinical assessment, and the potential disastrous consequences of inappropriate discharge. The role of OUs using clear diagnostic care pathways has shown that they can be more cost effective than referral to the in-hospital bed base for low to moderate risk chest pain. In the US, three randomised studies all identified lower costs using the ED Ous. $^{13} 1422$ A number of other descriptive "before and after" American studies have also suggested economic benefits with cost savings. ${ }^{23}$ Similar models have now also been shown to be effective and safe in the UK setting. ${ }^{11}{ }^{12}$

In the evaluation of another condition, Rydman et al studied the role of an ED based OU using an accelerated treatment protocol compared with standard inpatient medical care in the treatment of acute asthma. ${ }^{24}$ A total of 113 patients were randomised to the two arms and found to have lower costs with higher quality of life outcomes in those admitted to the OU. A subsequent patient satisfaction survey also revealed that the OU based group scored higher on four summary ratings. ${ }^{25}$ Other examples of the success of an OU based care pathway include the role of patients with abdominal pain. ${ }^{26} \mathrm{~A}$ descriptive study of 252 patients suggested benefit with a higher proportion of patients discharged home safely without the need for admission to the inhospital bed base and significantly reduced operative intervention.

The potential impact of a large multi-purpose OU has been more difficult to define and quantify. Martinez et al $^{27}$ described the throughput of their 12 bedded " 23 hour" OU over a three year period and then attempted to show the effect that it had in reducing the admission rate to hospital. The OU admitted patients with a range of $12-15$ conditions, the commonest being chest pain, asthma, cellulitis, substance misuse, abdominal pain, and diabetic emergencies. A total of 7507 patients were admitted to the OU at Cook County Hospital between 1996-1998 and 85\% were discharged home within 24 hours. A $12 \%$ reduction in admissions to the hospital bed base was achieved for a rate that had been previously steady. Unfortunately, the nature of the study design did not allow formal cost effectiveness evaluation. In a commentary of the paper, Ross and Zalenski ${ }^{21}$ pointed out that although high quality care could be delivered using an ED based observation unit model, the complex nature of the American healthcare reimbursement system is proving to be a disincentive to OU development at present. Essential to the success of these ED models are clear objectives and need for constant refinement. Further research is needed however to better define the impact that ED led service can have on the overall emergency care process.

\section{SETTING UP A CDU/OU}

Essential to the success of the CDU (OU) concept is the need for a clear vision shared among all staff of a system with tight structural organisation, processes of delivery and well defined outcome measures to evaluate performance. Structural organisation must encompass a clear strategy, operational policies, and specified critical pathways of care for individual groups of patients. Critical pathway techniques were first developed for use in industry to optimise production processes, minimise variability in practice and improve the quality of production. ${ }^{28}$ This in turn led to cost savings. Despite some potential limitations in the healthcare setting, Pearson $e a^{29}$ identified clear benefits to delivery in implementing this approach. In the US, developments in OU medicine have led to well defined policies for operational management as set out by the American College of Emergency Physicians and the Joint Commission of Accreditation of Healthcare Organizations. ${ }^{30}$

The size of a proposed unit can be difficult to define as it will depend on local practices, changing management strategies, and the move towards more ambulatory care. Existing OUs in the UK have been shown to have a workload of between $2 \%$ to $5 \%$ of the total number of attendances per year for a limited number of protocols. ${ }^{7}$ The existing BAEM guidelines ${ }^{6}$ of one bed/5000 ED is probably the most applicable if the unit is intended as multi-purpose and aims to maintain a bed occupancy rate of less than $85 \% .^{5}$ It should target patients with a range of low to moderate risk symptom complexes that with optimal diagnostic support could be discharged within a 6-24 hour period. Medical and nursing staff levels must be adequate to maintain a 24 hour seven day service. ${ }^{8}$

A well defined quality assurance programme is an essential component for success of the unit to monitor the processes of care with. This must describe among other things, patient volume, diagnosis, timeliness of care, admission rates to the in-hospital bed base, adverse events or deaths on the unit, complaints, unplanned re-attendance, and levels of follow up required. Staff must be empowered to be responsible for the quality of the data and learn to use them to develop their own areas of interest and expertise. Opportunites for good data capture and research are greatly increased in the more controlled environment of a CDU. ${ }^{27}$

\section{CONCLUSIONS}

The quality "bar" for delivering emergency medical care in the UK will continue to rise and be based not only on the timeliness of care but upon earlier diagnosis and appropriate need for admission to a valuable hospital bed. Performance targets in deciding on the need for hospital admission will need to be consistent, cost effective, and well balanced with a minimal likelihood of an adverse event or more importantly inappropriate discharge from the ED. These decisions are particularly difficult in patients with certain undifferentiated low to moderate risk symptom complexes who are treated in the ED.

The CDU concept provides one potential solution to deliver on this particular "stream" of patients. It requires enhanced working practices by staff and is well within the skillbase of the consultant in emergency medicine to develop, organise, and coordinate. Most importantly in some departments it will require managers, nurses, and doctors to make that elusive paradigm shift in thinking. Our experiences in the development of CDUs in Leeds have shown that benefits will accrue rapidly for patients, the ED staff (both medical and nursing) as well as the in-hospital specialties and their bed base. The units have become a core component of delivering quality care within our emergency care process as well as having refined the gate keeping systems within our hospitals. Further evaluation is ongoing to quantify the impact that the units have on this gate keeping role for patients with emergency care needs. If the specialty considers CDUs and observation medicine as a core function for the ED, well defined training programmes will need to be developed as part of the curriculum for trainees as well as position statements for standards of practice in the UK.

\section{Funding: none.}

Conflicts of interest: TBH is the Clinical Lead of the Leeds CDU Group and has been involved in developing the CDU concept in Leeds since September 2000. 


\section{REFERENCES}

1 Department of Health. The NHS Plan. London: Department of Health, 2001

2 Department of Health. Reforming emergency care. London: Department of Health, 2002. http://www.doh.gov.uk/ capacityplanning/reform.htm (accessed 10th October 2002)

3 Black D, Pearson M. Average length of stay, delayed discharge and hospital congestion. BM/ 2002:325:610-11.

4 NHS Confederation, Royal College of Physicians. Tackling emergency admissions:policy into practice. Birmingham: NHS Confederation, 1997

5 Bagust A, Place M, Posnett JW. Dynamics of bed use in accommodating emergency admissions: stochastic simulation model. BM 1999:319:155-8.

6 British Association for Accident and Emergency Medicine Clinical Services Committee. Recommendations for accident and emergency wards. London: BAEM, 1989

7 Goodacre SW. Role of the short stay observation ward in accident and emergency departments in the UK. J Accid Emerg Med 1998;15:26-30.

8 Ross MA, Graff LG. Principles of observation medicine. Em Med Clin N Am 2001;19:1-15

9 Dallos V, Mouzas GL. An evaluation of the functions of a short stay observation ward in the accident and emergency department. BM 1981;282:37-40.

10 Lewin W. Medical staffing and accident and emergency services. London: Joint Consultants Committee, 1978.

11 Herren KR, Mackway-Jones K, Richards CR, et al. Is it possible to exclude a diagnosis of myocardial damage within six hours of admission to an emergency department? Diagnostic cohort study. BM 2001;323:372.

12 Goodacre SW, Morris FM, Campbell S, et al. A prospective observational study of a chest pain observation unit in a British hospital. Emerg Med J 2002;19:117-121.

13 Farkouh ME, Smars PA, Reeder GS, et al. A clinical trial of a chest pain observation unit for patients with unstable angina. N Engl J Med 1998;339: 1882-8

14 Roberts RR, Zalenski RJ, Mensah EK, et al. Costs of an emergency department based accelerated diagnostic protocol vs hospitalisation in patients with chest pain. A randomised controlled trial. JAMA 1997;278: 1670-6

15 Landers FA, Waeckerle JF, McNabney WK. Observation ward utilization. Journal of the American College of Emergency Physicians $1975 ; 4: 123-5$
16 Diamond NJ, Schofferman JA, Elliott JW. Evaluation of an emergency department observation ward. Journal of the American College of Emergency Physicians 1976;5:29-31.

17 American College of Emergency Physicians. Emergency department observation units. Ann Emerg Med 1988;17:95-6.

18 Graff L, Zun J, Leikin S, et al. Emergency department observation beds improve patient care. Society for academic emergency medicine debate. Ann Emerg Med 1998;21:967-75.

19 Observation Medicine Committee, SAEM Observation medicine curriculum. Ann Emerg Med 1992;21:963-96.

20 Counselman FI, Schafermeyer RW, Garcia R, et al. A survey of academic departments of emergency medicine regarding operation and clinical practice. Ann Emerg Med 2000;36:446-50.

21 Ross MA, Zalenski RJ. Observation services - past, present and future. Am J Med 2001;1 10:324-5.

22 Gomez MA, Jeffrey LA, Karagounis LA, et al. An emergency department based protocol for rapidly ruling out myocardial ischaemia reduces hospital time and expense: results of a randomised study (ROMIO). J Am Coll Cardiol 1996;28:25-33.

23 Roberts R, Graff LG. Economic issues in observation unit medicine. Emerg Med Clin North Am 2001;19:19-31.

24 Rydman RJ, Isola ML, Roberts RR, et al. Emergency department observation unit versus hospital inpatient care for a chronic asthmatic population. Med Care 1998;36:599-609.

25 Rydman RJ, Roberts RR, Albrecht GL, et al. Patient satisfaction with an emergency department asthma observation unit. Acad Emerg Med 1999;6:178-83.

26 Graff LG, Radford M, Werne C. Probability of appendicitis before and after observation. Ann Emerg Med 1991;20:503-7.

27 Martinez E, Reilly BM, Evans AT, et al. The observation unit: a new interface between inpatient and outpatient care. Am J Med $2001 ; 110: 274-7$

28 Greene JH. Production and inventory control: systems and decisions. Homewood, IL: Ricahrd D Irwin, 1974.

29 Pearson SD, Goulart-Fisher D, Lee TH. Critical pathways as a strategy for improving care: problems and potential. Ann Intern Med 1995; 123:941-8.

30 Brillman L, Mathers-Dunbar L, Graff L, et al. Management of observation units. Ann Emerg Med 1995;25:823-30. 